\title{
The Abdominal Muscles - a Functional Review
}

\begin{abstract}
The role of the abdominal muscles in human function as well as their physiological mechanisms for these has been the subject of an increasing volume of reported research for more than 20 years. The abdominal muscles participate in a wide range of diverse functional tasks. These include breathing, postural stability, expulsive maneuvers and speech control. To perform these functions effectively and often concurrently, based on demand, the muscles are anatomically, physiologically and biochemical suited. The idea that the abdominal muscles function as a unit has been dispelled by many scientific reports. It is now known that they function differentially as individual muscles and also differentially at the motor unit level. Regional differences in the activation patterns of these muscles are also known. In addition the motor units within muscles are functionally organized to either subserve a specific function or more than one function. Knowledge of the functional organization of the abdominal muscles is critical to any rehabilitation process especially since rehabilitation is holistic and strives to achieve functional goals. This functional review is based on current scientific evidence for the multi functionality of all abdominal muscles. Literature based on animal studies has been excluded as far as is possible.
\end{abstract}

KEY WORDS: FUNCTIONAL REVIEW, ABDOMINAL MUSCLES, BREATHING, POSTURE.

\section{INTRODUCTION}

The abdominal muscles are multifunctional in nature (Goldman et al 1987). The anatomical, neurophysiological, biochemical and morphological features of the abdominal muscles makes them ideally suited to perform multiple functions efficiently. The neurophysiology of this multifunctionality has only recently been elucidated (Puckree et al 1998). As will be shown from the literature a complex interaction of respiratory and postural demands on the abdominal muscles help to optimize respiration and trunk control not only during rapid arm movements (Hodges et al 1997a,b) but also during any task.

The abdominal muscles are primarily or secondarily involved in stability and mobility and a combination of the two. This is supported by the histochemical and metabolic make up, namely the presence of types $1,11 \mathrm{a}$, and $\mathrm{b}$ and $\mathrm{c}$ fibers. In addition, the fact that some muscles are attached to the skeleton and

\section{CORRESPONDENCE TO:}

\section{Prof. T. Puckree}

Department of Physiotherapy,

University of KwaZulu Natal,

Private Bag X54001, Durban, 4000

Tel: (031) 260-7977

Fax: (031) 260-8106

E-Mail: puckreet@ukzn.ac.za others to fascia makes the abdominals unique in their functions. The abdominals may function as a unit when required and individually based on demand. Some of the unique characteristics of the abdominal muscles namely regional and differential activation, and functional organization of motoneuron pools will help understand these muscles from a functional standpoint. When specific muscles were the basis of research in the studies cited, these are mentioned.

Regional differences in the activity of the abdominal muscles during respiratory and non-respiratory tasks have been reported (Strohl et al 1981, Floyd and Silver 1950). Goldman and Silver (1987) reported differences in upper and lower abdominal muscle activity during postural changes. In standing, tonic electromyographic (EMG) activity was relatively greater in the lower abdomen than in the upper. This activity was abolished by the assumption of the supine posture or voluntary relaxation (Strohl et al 1981, Hodges et al, 1997). Relative activity of the upper and lower abdomen was equal during speech, recitation of verse, cough or laughter when studies were conducted using indwelling electrodes. Regional differences in the participation of abdominal muscles during speech were reported by some investigators (Hoit et al 1988) who used surface electrodes. Postural events utilize mainly the lower abdomen while breathing uses mainly the upper abdomen (Strohl et al 1981). During sit ups and curl ups, researchers found greater activity in upper rectus abdominus (RA) (Sarti et al, 1996) compared to greater activity in the lower RA during pelvic tilt and leg raising exercises (Flint and Gudgell 1965). Lehman and McGill (2001) reported no differences in the responses of the upper and lower RA to curl ups, head and shoulders raise and leg raises. The external abdominal oblique (EO) showed regional differences in response to the same exercises (Lehman and McGill 2001). The differences in the scientific findings presented above could be attributed to whether the investigators used indwelling electrodes or surface electrodes and also to individual variation in abdominal muscles responses.

\section{Differential action}

Traditionally the abdominal muscles have been considered to function as a single unit during breathing and other tasks (Floyd and Silver, 1950). The differential action of individual abdominal muscles is now a fact (Abe et al 1996, Hodges et al 1997, Strohl et al 1981, Puckree et al 1998). Differential activation of the abdominal muscles is related to the different proportions of muscle lengthening (Abe et al 1996). It has also been observed that the abdominal 
muscles lengthen in a particular order in response to increasing lung volume namely, TA, Internal abdominal oblique (IO), EO and RA. Therefore differential activation also occurs in this order. The same order has also been observed with postural change or chemical stimulation (Abe et al 1996). It was also found that the abdominal muscles lengthen most at end inspiration.

The location, attachments and differing fiber orientation of the individual abdominal muscles may predict their differing response to posture or chemical stimulation. Leevers and Road (1994) who based on their work on canines believe that segmental reflexes have a significant influence on the differential recruitment of the abdominal muscles. The RA, which on contraction brings the xiphisternum closer to the pubis, is likely to provide more deflation of the abdomen compared to the other three muscles. The innermost TA which extends circumferentially, on contraction will contrict the abdominal contents directly. The fact that this muscle does not have any direct bony attachments makes it more effective mechanically in increasing abdominal pressure and influencing the length of the diaphragm (Abe et al 1996).

\section{Functional organization of motor neuron pools.}

Puckree et al (1997) showed that the motoneuron pools of the TA, IO and TA are functionally organized into task groups and micro groups. During the performance of natural tasks, e.g., quiet breathing, leg lifting in standing (simulating a natural step forward) and loaded expiration, different groups of IO and TA motor units were recruited for each task with little overlap between groups. Those motor neurons which became active in more than one task showed distinct recruitment and discharge patterns. This suggests that some motor units from a specific muscle will subserve only one function while other motor units will subserve more than one function. This study showed that recruitment and rate coding of abdominal motorneurons were phase linked to the respiratory cycle regardless of what the task was. Their activity during the respiratory cycle affects their reaction times for postural tasks (Hodges et al 1997).

\section{ROLE OF ABDOMINAL MUSCLES IN RESPIRATION:}

\section{Quiet breathing:}

Traditionally, it was believed that expiration is a passive process with the abdominal muscles becoming active only during voluntary expiration. While the former has been challenged, the latter has been supported. In supine resting humans, Abe et al (1996) showed significant phasic electromyographic (EMG) activity of the TA during expiration. A change in position from supine to standing showed the greatest activity in the TA with lesser activity of the IO and EO while RA remained inactive. These findings suggest that resting expiration is active and that abdominal muscles are activated differentially.

Abdominal muscles are potent muscles of expiration while acting as accessory muscles to inspiration (Abe et al 1996). Despite evidence for this, some investigators have found that EMG activity of the abdominal muscles is rarely recorded during quiet breathing (Hodges et al 1997). The latter was observed only when the subject was completely relaxed with the use of biofeedback. However when ventilation is increased as occurs during exercise the abdominal muscles are recruited toward the end of expiration (Goldman et al 1987). This contraction of the RA, EO, IO and TA increases expiration below functional residual capacity to increase inspiratory volume when ventilation is increased voluntarily (De Troyer et al 1990, Goldman et al 1987).

The coordinated use of the abdominal muscles facilitates inspiration by 2 mechanisms (De Troyer and Loring 1986; Loring and De Troyer, 1985). 1. Increased expiratory flow reduces end expiratory lung volume thereby prolonging expiration. The reduction of lung volume below passive end expiratory volume induces inspiratory elastic pressure. 2. The pressure generating capacity of the diaphragm is increased as the diaphragm is elongated by expiratory muscle action. Contraction of the abdominal muscles raises abdominal pressure which displaces the diaphragm into the thorax and thus lengthens diaphragmatic fibers and places them on a more advantageous portion of the length tension curve (Abe et al 1996)
Effect on the diaphragm: The abdominal muscles help to regulate the length of the diaphragm, (De Troyer 1983, Abe et al 1996). The abdominal muscles compress the abdominal contents, thereby displacing the diaphragm into the thorax, and decreasing lung volume. As such they are considered to be potent expiratory muscles especially during stimulated ventilation as occurs during exercise, $\mathrm{CO}_{2}$ rebreathing and loaded inspiration.

Action of the abdominal muscles on the rib cage: The abdominal muscles are thought to pull the lower ribs down and deflate the rib cage. Indirectly these muscles by increasing intra abdominal pressure cause passive distension of the diaphragm which by acting over its zone of apposition to the lower rib cage expands this part of the rib cage (Mier et al, 1985). The RA and EO each act differently on the rib cage. By increasing the transverse diameter of the rib cage, the RA pulls the rib cage caudally and thereby decreases the rib cage anteroposterior diameter. It makes the rib cage more elliptical. The EO contracts the lower rib cage along its transverse diameter making it more cylindrical. When activated together, the distortion of the rib cage will depend on the relative strength of contraction of each of the muscles. The IO and TA were not tested (Mier et al, 1985).

\section{Loaded breathing:}

When ventilation is stimulated e.g. during exercise, and $\mathrm{CO}_{2}$ stimulation, the abdominal muscles contribute to the inspiratory pump action (Abe et al 1996). Takasaki et al (1989) reported a progressive response of EO to progressive hypercapnia with hyperoxia while Abe et al (1996) showed progressive increase in phasic activity of all 4 abdominal muscles during hperoxic progressive hypercapnia. On the other hand, hypercapnic or loaded ventilation elicit recruitment of TA and IO at lower minute ventilation than the other abdominal muscles (De Troyer et al 1990, Wakai et al 1992). Loading of inspiration elicited activity throughout expiration in the TA and the IO only, similar to the findings of De Troyer et al (1990). However when expiration is forced below Functional Residual 
Capacity (FRC) all abdominal muscles became active. Hodges et al (1997) showed that the abdominal muscles increased their activity during inspiratory loading (breathing through a narrow tube of specified diameter and length), forced expiration below FRC and a static glottis-closed expulsive maneuver.

During exercise, the abdominal muscles play a supporting role in determining the ventilatory response (Abraham et al 2002). Increases in blood $\mathrm{CO}_{2}$ concentrations (Abe et al 1996), end expiratory lung volume (Henke et al 1988, Bishop 1974) and increased expiratory flow resistance (Baker et al 1979) potently activate the abdominal muscles.

\section{ROLE OF ABDOMINAL MUSCLES IN NON-RESPIRATORY TASKS.}

The non-respiratory tasks in which the abdominal muscles play an important role include posture control, stability of the trunk, and movements of the trunk and limbs, speech, and expulsion and coughing.

\section{Posture}

The abdominal muscles contribute to the maintenance of postural equilibrium and stabilization of the trunk (Aruin and Latash 1995, Cresswell et al 1992, Hodges and Richardson 1997). Contraction of the anterior abdominal muscles occurs as part of postural stabilization before movement of the upper/lower limb (Hodges and Richardson 1997 a,b) or voluntary loading of the trunk (Cresswell et al 1994). When rapid movements of the arm/leg produce brief challenges to postural stability of the trunk, the TA contracts prior to the limb muscles whereas the IO, EO and RA contract after the limb muscles responsible for initiation of the movements (Hodges and Richardson 1997 a,b). In order for movements of the limbs to occur, postural stability is required, subserved by the abdominal muscles (Hodges and Richardson 1997 a,b). Electrophysiologically, it has been shown that the abdominal muscles are depolarized during expiration and hyperpolarized in inspiration. This implies that the latency to onset of abdominal muscles contraction for postural control is shorter when the motoneuron pool is already recruited. In the relaxed standing position, Hodges et al
(1997) reported a "silence" in the electromyograms of the abdominal muscles in contrast to the results of other investigators (De Troyer 1983, Floyd and Silver 1950, Strohl et al 1981). Hodges et al (1987) used biofeedback to get their subjects to relax, hence their findings. The contribution of the individual abdominal muscles to postural control is influenced by their role in respiration (Hodges et al 1997, Puckree et al 1998).

When arm movements began in expiration, the onset of EMG activity in the TA and IO relative to that of the deltoid muscle was significantly earlier compared to that during inspiration (Hodges et al 1997). Although the recruitment and rate coding of abdominal IO and TA motor units showed task specificity, or multifunctionality, their activation was always phase linked to the respiratory cycle (Puckree et al 1998).

\section{Trunk Movements}

The abdominal muscles play an important role in trunk stability and movement, differentially. Cresswell et al (1992) demonstrated that the TA contracts continuously during lumbar spine extension and flexion movements possibly fulfilling its stabilizing function. The TA was found to be the first muscle to contract during trunk movements (Hodges 1999) supporting its primary role as a stabilizer of the spine. It has also been reported that the TA acts independently of the other abdominal muscles to protect the lumbar spine against potentially damaging forces (Cresswell et al, 1992; Hodges et al 1996, Hodges 1999). In flexing the trunk with the knees bent, the abdominal muscles are active between 30-45 degrees i.e. at the beginning of the movement (Moraes et al, 2003). From the erect position contraction of the RA is not required for spinal flexion due to the location of the center of gravity in either the sitting or standing postures (Ekholm et al, 1979). Rotation of the trunk elicited large amounts of activity from the EO with little or no activity from the RA or TA. The RA is an antagonist to the longitudinal parts of the erector spinae system. It may play an important role in preventing overloading the spine (Floyd and Silver 1950; Partridge and Walters 1959). De Troyer et al (1990) showed that flexing the head against a resistance elicited large amounts of activity from the RA with little or no activity from the EO.

\section{Limb movements}

Hodges et al a,b (1997) showed that the abdominal muscles contract before agonist limb muscles when limbs are moved by a standing subject. It has also been shown that the TA contracts in preparation for reaction time movements of the arm (Hodges et al 1997a) or leg (Hodges et al 1997b). The preparatory contraction of the abdominal muscles may increase intra-abdominal pressure to assist in the stabilization of the trunk and control postural equilibrium disturbed by movement of the arm (Cresswell et al 1994, Hodges and Richardson 1997). This may be due to the increase in tension of the thoracolumbar fascia through which TA attaches to the spine (Tesh et al 1987) plus the increase in intra-abdominal pressure (Cresswell et al 1992). Hodges and Richardson a(1997) suggest that the abdominal muscles prepare the body for unexpected disturbance to postural equilibrium and spinal stability provoked by reactive forces resulting from limb movement.

\section{Expulsive maneuvers:}

De Troyer et al (1997), reported large amounts of EMG activity from the TA, RA and EO during expulsive maneuvers with the glottis closed. Static expulsive maneuvers elicited preparatory contractions that are involved in stabilizing the trunk (Hodges et al 1997). TA and IO are believed to play a more important role in mechanical stabilization of the trunk (Cresswell et al, 1992, Cresswell et al 1994)

\section{Coughing counting, speaking, laughing.}

Both TA, RA and EO (to a lesser extent) were active during these tasks (De Troyer et al, 1990). The RA is not active during coughing (Floyd and Silver 1950; Partridge and Walters 1959).

\section{SUMMARY}

This concise review provides evidence for the multifunctionality, differential and regional differences in activation patterns of individual abdominal muscles and the functional organization of abdominal motor units. It shows that the abdominal muscles are potent expiratory 
muscles while serving a very important accessory role during inspiration. From a functional standpoint it becomes clear that the abdominal muscles are important in a range of tasks. Task specificity of pools of motoneurons suggests specificity in strengthening during rehabilitation. On the other hand those pools that receive more than one type of input can benefit from the imposition of more than one functional demand at the same time for example, a weak patient is encouraged to coordinate breathing with walking and postural control.

\section{REFERENCES}

Abe T, Kusushara N, Yoshimura N, Tomita T, Easton PA. 1996 Differential respiratory activity of four abdominal muscles in humans. Journal of applied Physiology 80(4): 1379-89.

Abraham KA, Feingold H, Fuller DD, Jenkins M, Mateika JH, Fregosi RF. 2002 Respiratory related activation of human abdominal muscles during exercise. Journal of Physiology 541(2): 653-663

Agostone E and Campbell EJM 1970 The Abdominal muscles. In: The respiratory muscles: Mechanisms and neural control ed. EJM Campbell, E. Agostoni and J Newsom-Davis. London: Lloyd-Luke, pp 175-180

Aruin AS and ML Latash 1995 Directional specificity of postural muscles in feed-forward postural reactions during fast voluntary arm movements. Experimental Brain Research 103: 323-332

Baker JP, Frazier DT, Hanley M, Zechman FW 1979 Behavior of expiratory neurons in response to mechanical and chemical loading. Respiration Physiology 36: 337-351

Bishop B 1974 Abdominal muscle activity during respiration. In: Ventilatory and Phonatory control system. Ed BD Wyke. Oxford University Press, Oxford, pp 12-24.

Cresswell AG, H. Grundstrom, A Thorstensson. 1992 Observations on intra-abdominal pressure and patterns of intra-abdominal pressure in man Acta Physiologica Scandinavia 144: 409-418

Cresswell AG, Oddsson L, Torstensson A. 1994 The influence of sudden perturbations on trunk muscle activity and intra-abdominal pressure while standing. Experimental Brain Research 98: 336-341

De Troyer A 1983 Mechanical role of the abdominal muscles in relation to posture Respiration Physiology 53: 341-353
De Troyer A, Estenne M, Ninane V, Van Gansbeke D, Gorini M. 1990 Transversus abdominus muscle function in humans. Journal of applied Physiology 68: 1010-1016

De Troyer A, Loring SH 1986. Action of the respiratory muscles. In: Handbook of Physiology. The respiratory system. Mechanics of breathing. Bethesda. MD: American Physiological Society. Sec. 3, vol III, pt 2, p443-461.

Floyd WF, Silver PHS. 1950 Electromyographic study of patterns of activity of the anterior abdominal wall muscles in man. Journal Anatomy 84: 132-145

Ekholm J, Arborelius U, Fahlcrantz A, Larsson A_M, Mattsson G. 1979 Activation of abdominal muscles during some physiotherapeutic Exercises. Scandinavian Journal of Rehabilitation Medicine. 11: 75-84

Flint MM, Gudgell J. 1965 Electromyographic study of abdominal muscular activity during exercise. Research Quarterly 36: 29-37

Goldman JM, Lehr RP, Millar AB , Silver JR. 1987 An electromyographic study of the abdominal muscles during postural and respiratory maneuvers. Journal of Neurology Neurosurgery Psychiatry 50: 866-869

Henke K G, Sharratt M, Pegelow D, Dempsey JA. 1988 Regulation of end-expiratory lung volume during exercise. Journal of Applied Physiology 64: 135-146

aHodges PW, Richardson CA 1997 Feedforward contraction of transverses abdominus is not influenced by the direction of arm movement. Experimental Brain Research, 114(2): 362-70

bHodges PW, Richardson CA 1997 Contraction of the Abdominal muscles Associated with movement of the lower limb Physical Therapy, 77(2): 132-141

Hodges PW 1999 Is there a role for transverses abdominus in lumbo-pelvic stability? Manual Therapy 4: 74-86

Hodges PW, Richardson C, Jull G. 1996 Evaluation of the relationship between laboratory and clinical tests of transverses abdominus function Physiotherapy Research International 1(1): 30-40

Hodges PW, Gandevia SC, Richardson CA 1997 Contractions of specific abdominal muscles in postural tasks are affected by respiratory maneuvers. Journal of Applied Physiology 83(3):753-760

Hoit JD, Plassman BL, Lansing RW, Hixon TJ 1988 Abdominal muscle activity during speech production. Journal of Applied Physiology 65(6):2656-2664
Leevers AM, Road JD 1989 Mechanical response to hyperinflation of the two abdominal muscle layers. Journal of Applied Physiology, $66: 2189-2195$

Leevers AM, Road JD 1994 Abdominal muscle activity during hypercapnia in awake dogs. Journal of Applied Physiology, 77 :1393-1398

Lehman GJ, McGill SM 2001 Quantification of the differences in electromyographic Activity magnitude between the upper and lower portions of the Rectus Abdominus muscle during selected trunk exercises. Physical Therapy 81(5): 1096-1101.

Loring SH , De Troyer A. 1985. Action of the respiratory muscles. In: The Thorax, ed. C Roussos and PT Macklem. New York: Dekker. vol 29, pt A, chapter 10, p 327-349.

Mier M, Brophy C, Estenne M, Moxham J, Green M, De Troyer A. 1985.Action of abdominal muscles on rib cage in humans. Journal of applied Physiology 58(5):1438-1443.

Moraes AC, Bankoff ADP, Almeida TL, Simoes EC, Rodrigues CEB, Okano AH. 2003. Using weights in abdominal exercises: electromyographic response of the rectus abdominus and the rectus femoris muscle. Electromyography and clinical Neurophysiology 43: 487-496.

Partridge MI, Walter CE 1959 Participation of abdominal muscles in various movements of the trunk in man: an emg-study. Physical Therapy Reviews. 39: 791-800

Puckree T, Cerny F, Bishop B 1998 Abdominal mot unit activity during respiratory and non-respiratory tasks. Journal of Applied Physiology 84(5): 1707-1715

Sarti MA, Monfort M, Fuster MA, Villaplana LA. 1996. Muscle activity in upper and Lower Recus abdominus during Abdominal exercises. Archives of Physical Medicine and Rehabilitation. 77: 193-7

Strohl KP, J Mead, B Banzett, SH Loring, PC Kosch 1981 Regional differences in abdominal muscle activity during various maneuvers in humans. Journal of Applied Physiology 51: 1471-1476

Takasaki Y, Orr D, Popkin J, Xie A, Bradley TD. 1989 Effect of hypercapnia and hypoxia on respiratory muscle activation in humans. Journal of Applied Physiology 67: 1776-1784

Tesh KM, ShawDunn J, Evans JH. 1987 The abdominal muscles and vertebral stability. Spine 12: 501-508

Wakai Y, Welsh MM, Leevers AM, Road JD. 1992 Expiratory muscle activity in the awake and sleeping human during lung inflation and hypercapnia. Journal of Applied Physiology 72:881-887 